\title{
Modifications of midpalatal sutural density induced by rapid maxillary expansion: A low-dose computed-tomography evaluation
}

\author{
Lorenzo Franchi, ${ }^{\text {a }}$ Tiziano Baccetti, ${ }^{\text {a }}$ Roberta Lione, ${ }^{b}$ Ezio Fanucci, ${ }^{c}$ and Paola Cozza ${ }^{d}$ \\ Florence and Rome, Italy
}

\begin{abstract}
Introduction: The aim of this study was to evaluate the density of the midpalatal suture as assessed by lowdose computed tomography (CT) before rapid maxillary expansion (RME) (TO), at the end of active expansion (T1), and after a retention period of 6 months (T2). Methods: The study sample comprised 17 prepubertal subjects (mean age, 11.2 years) with constricted maxillary arches and unilateral or bilateral posterior crossbite. The total amount of expansion was $7 \mathrm{~mm}$ in all subjects. Multi-slice low-dose CT scans were taken at T0, T1, and T2. On axial CT scanned images, 4 regions of interest (ROIs) were placed along the midpalatal suture (anterior [AS ROI] and posterior [PS ROI]) and in 2 regions of palatal bone (anterior and posterior). Density was measured in Hounsfield units. The Mann-Whitney $U$ test and Friedman analysis of variance (ANOVA) with posthoc test were used $(P<0.05)$. Results: The densities in the AS and PS ROls were significantly smaller than the reference bone densities before RME therapy. Both AS and PS ROls showed significant decreases in density from T0 to T1, significant increases from T1 to T2, and no significant differences from T0 to T2. Conclusions: The effective opening of the midpalatal suture by RME in prepubertal subjects was associated with a significant decrease in sutural density. The sutural density after 6 months of retention post-RME indicated reorganization of the midpalatal suture, since it showed values similar to the pretreatment ones. (Am J Orthod Dentofacial Orthop 2010;137:486-8)
\end{abstract}

$\mathbf{R}$ apid maxillary expansion (RME) is an effective orthopedic procedure commonly used to treat maxillary transverse deficiencies in growing patients. ${ }^{1}$ Transverse dentoskeletal changes in the maxilla in response to RME are produced mainly through disruption of the midpalatal suture. ${ }^{2}$ Histologic studies on both humans and animals supported the opening of the midpalatal suture after RME therapy. ${ }^{3,4}$ In a study of 6 monkeys, Cleall et $\mathrm{al}^{3}$ reported that, at the moment of suture opening, the area was filled with unorganized fibrous conjunctive tissue; 6 months after expansion, the

\footnotetext{
${ }^{a}$ Assistant professor, Department of Orthodontics, University of Florence, Florence, Italy; Thomas M. Graber Visiting Scholar, Department of Orthodontics and Pediatric Dentistry, School of Dentistry, University of Michigan, Ann Arbor.

besearch fellow, Department of Orthodontics, University of Rome "Tor Vergata," Rome, Italy.

cProfessor, Department of Radiology, University of Rome "Tor Vergata," Rome, Italy.

${ }^{\mathrm{d}}$ Professor and chair, Department of Orthodontics, University of Rome "Tor Vergata," Rome, Italy.

The authors report no commercial, proprietary, or financial interest in the products or companies described in this article.

Reprint requests to: Tiziano Baccetti, Department of Orthodontics, University of Florence, Via del Ponte di Mezzo, 46-48, Florence, Italy 50127; e-mail, t.baccetti@odonto.unifi.it.

Submitted, July 2009; revised and accepted, October 2009. $0889-5406 / \$ 36.00$

Copyright (C) 2010 by the American Association of Orthodontists.

doi:10.1016/j.ajodo.2009.10.028
}

suture was well organized and appeared histologically normal.

Dimensional changes in the midpalatal suture produced by RME in growing subjects have been investigated by conventional radiographic techniques such as posteroanterior cephalograms ${ }^{2,5}$ and occlusal radiographs. ${ }^{6}$ Recently, the opening of the midpalatal suture after RME was evaluated with computed tomography (CT), which provides accurate 3-dimensional assessment of skeletal changes. ${ }^{7-9}$ The use of CT has been proposed also for the quantitative evaluation of bone density with the Hounsfield index in selected regions of interest (ROI). ${ }^{10}$ The same index has been used to assess bone density for implant placement in the maxillary bones. ${ }^{11}$

The aim of this study was to evaluate treatment and posttreatment changes produced by RME in the density of the midpalatal suture with a low-dose CT protocol.

\section{MATERIAL AND METHODS}

The prospective study sample comprised 17 white subjects ( 7 boys, 10 girls) with a mean age of 11.2 years (range, 8-14 years) who sought orthodontic treatment at the Department of Orthodontics of the University of Rome "Tor Vergata." Criteria for enrollment in the study were as follows: constricted maxillary arches with or without unilateral or bilateral posterior 

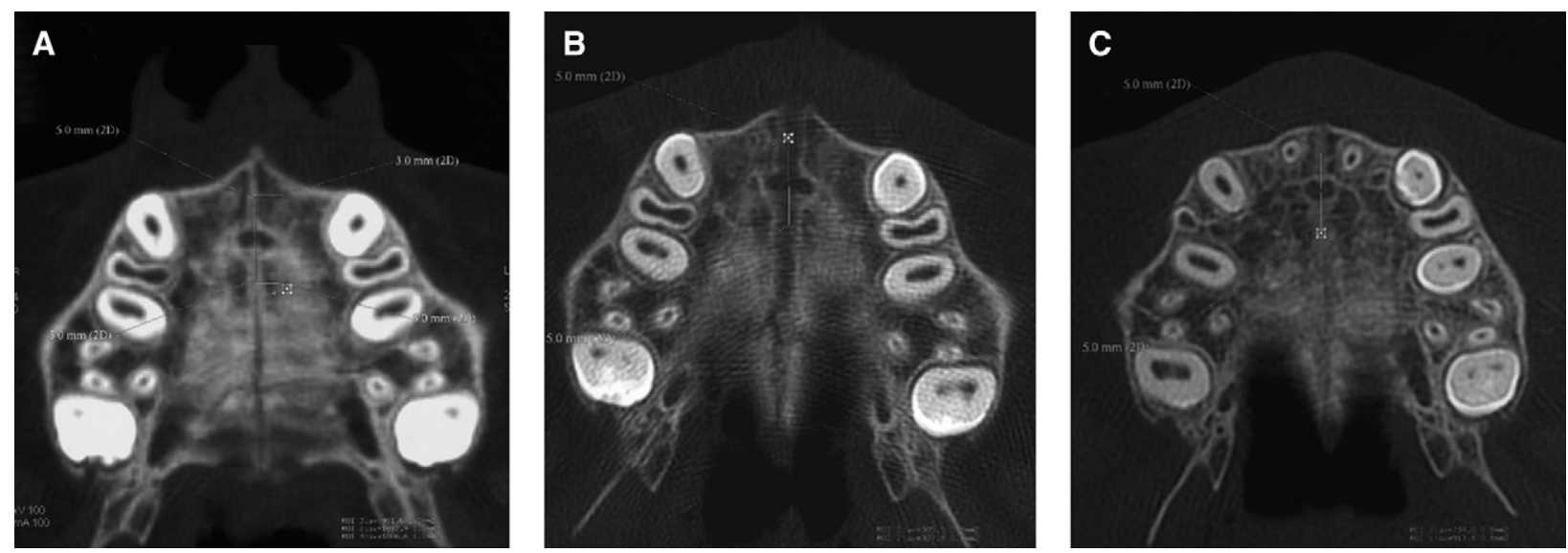

Fig. Axial scans taken A, before RME, B, at the end of the active expansion phase, and $\mathbf{C}$, after retention of 6 months, with the 4 ROls where density was recorded. The numbers indicate where the ROls were located at the 3 observation points.

crossbite, and prepubertal stages of cervical vertebral maturation (CS1-CS3) as assessed on lateral cephalograms. ${ }^{12}$ This project was approved by the ethical committee, and informed consent was obtained from the patients'parents.

Each patient underwent a standardized protocol of RME with the butterfly palatal expander that followed the basic design of Haas. ${ }^{13}$ The expansion screw was activated twice per day $(0.25 \mathrm{~mm}$ per turn $)$ for 14 days, for a total amount of screw expansion of $7 \mathrm{~mm}$ in all subjects. Then the screw was tied off with a ligature wire, and the expander was kept in place as a passive retainer for 6 months.

Multi-slice low-dose CT scans were taken before rapid palatal expansion (T0), at the end of the active expansion phase (T1), and after the retention period of 6 months (T2). The low-dose CT scan protocol was described previously. ${ }^{14}$ Standardized axial CT images parallel to the palatal plane and passing through trifurcation of the maxillary right first molar were acquired and enlarged by a 3-times magnification factor with specific software (Light-Speed 16, General Electric Medical System, Milwaukee, Wis). On the enlarged images, 4 ROIs that extended to an area of $1 \mathrm{~mm}^{2}$ were placed by 1 trained operator (R.L.) for the calculation of values of density in Hounsfield units (HU) (Fig). The operator was blinded to the patient being measured. The 4 ROIs in the palatal region were defined as follows.

1. Anterior sutural ROI (AS ROI): density values measured in the ROI located along the midpalatal suture $5 \mathrm{~mm}$ in front of the center of the nasopalatine duct at T0, T1, and T2.

2. Posterior sutural ROI (PS ROI): density values measured in the ROI located along midpalatal suture $5 \mathrm{~mm}$ posterior to the center of the nasopalatine duct at $\mathrm{T} 0, \mathrm{~T} 1$, and $\mathrm{T} 2$.

3. Anterior bony ROI (AB ROI): density values measured in the ROI located on the palatal bone $3 \mathrm{~mm}$ laterally (on the right side) to the AS ROI at T0.

4. Posterior bony ROI (PB ROI): density values measured in the ROI located on the palatal bone $3 \mathrm{~mm}$ laterally (on the right side) to the PS ROI at T0.

The last 2 values were used as references to compare the density of the midpalatal suture with that of the bony palate.

\section{Statistical analysis}

One operator (R.L.) performed all measurements at the same scanner console and repeated all measurements 1 month later. Systematic and random errors of the 4 measurements of the 17 subjects at all observations periods were calculated with paired $t$ tests and Dahlberg's formula, ${ }^{15}$ respectively. The Mann-Whitney $\mathrm{U}$ test was used to compare the density between the sutural ROIs and the bony ROIs at T0, and between the anterior and posterior sutural ROIs at the 3 time points. The changes in density from T0 through $\mathrm{T} 2$ both in the anterior and posterior sutural ROIs were contrasted with the Friedman repeated measures ANOVA on ranks followed by the Tukey post-hoc test (SigmaStat 3.5, Systat Software, Point Richmond, Calif). The level of significance was set at $P<0.05$.

\section{RESULTS}

No systematic errors for any of the 4 density measures at the different observation periods were found 
$(P>0.05)$. Random errors for density ranged from 0.17 at the PS ROI at T1 to 0.40 at the PS ROI at T0. The values of bone density in the AS ROI and the PS ROI at $\mathrm{T} 0(563.3 \pm 183.2 \mathrm{HU}$ and $741.7 \pm 167.1 \mathrm{HU}$, respectively) were significantly smaller than those in the $\mathrm{AB}$ ROI and the PB ROI at T0 $(1057.5 \pm 129.4 \mathrm{HU}$ and $1102.8 \pm 160.9 \mathrm{HU}$, respectively). The density in the AS ROI was significantly smaller than in the PS $\mathrm{ROI}$ at $\mathrm{T} 0$, but no significant differences were recorded between the 2 sutural ROIs at T1 and at T2. Both AS and PS ROIs showed significant decreases in density from $\mathrm{T} 0$ to $\mathrm{T} 1$, significant increases from $\mathrm{T} 1$ to $\mathrm{T} 2$, and no statistically significant differences from $\mathrm{T} 0$ to $\mathrm{T} 2$.

\section{DISCUSSION}

We applied low-dose CT to the evaluation of the changes in density produced by RME in the midpalatal suture after active expansion and after 6 months of retention. CT has been used in oral implantology to assess bone density for a proposed implant site. ${ }^{10,11}$ However, no previous studies with CT scans to evaluate the density of craniofacial sutures in growing subjects were found.

The gray level values recorded in the AS ROI before RME were significantly smaller than the values measured in the PS ROI. This difference in density explains the greater opening in the anterior vs the posterior sutural regions after the active phase of expansion, as demonstrated in a previous study. ${ }^{9}$ Lione et $\mathrm{al}^{9}$ reported that the amount of transverse increase at the middle sutural width (corresponding to PS ROI in our study) was about $70 \%$ of that observed in the anterior sutural width (corresponding to AS ROI in our study).

The gray level values of the midpalatal suture before RME were significantly smaller than those in the palatal bone, both anteriorly and posteriorly. Sutural densities in the AS ROI and the PS ROI were about 50\% and $30 \%$ smaller, respectively, than the corresponding palatal bone ROIs. This finding indicates that, at a prepuberal stage of skeletal development, the midpalatal suture has not completed its ossification. RME produced a sutural opening in all patients of our sample that was confirmed by a reduction of density after active expansion. The value of density at $\mathrm{T} 1$ was $70 \%$ smaller than the value at T0. After the 6-month retention period, the midpalatal suture appeared to be reorganized with density similar to the value at $\mathrm{T} 0$. This confirms previous histologic observations indicating that a retention period of 6 months with the RME in place allows for reorganization of the suture. ${ }^{3}$ In future investigations, it would be interesting to test whether the density of the midpalatal suture tends to increase in postpubertal subjects and whether a different density correlates with a different amount of suture opening. Low-dose CT could then provide information about individual responsiveness of the midpalatal suture to RME therapy.

\section{CONCLUSIONS}

1. In prepubertal subjects, the density of the midpalatal suture is less than the density measured in the maxillary bone.

2. The reduced sutural density after expansion indicates that RME therapy before puberty produces effective opening of the midpalatal suture.

3. Six months of retention after RME allows reorganization of the midpalatal suture with density values similar to the pretreatment ones.

\section{REFERENCES}

1. McNamara JA. Maxillary transverse deficiency. Am J Orthod Dentofacial Orthop 2000;117:567-70.

2. Wertz RA. Skeletal and dental changes accompanying rapid midpalatal suture opening. Am J Orthod 1970;58:41-66.

3. Cleall JF, Bayne DI, Posen JM, Subtelny JD. Expansion of the midpalatal suture in the monkey. Angle Orthod 1965;35:23-35.

4. Melsen B. Palatal growth studied on human autopsy material. A histologic microradiographic study. Am J Orthod 1975;68:42-54.

5. Cameron CG, Franchi L, Baccetti T, McNamara JA Jr. Long-term effects of rapid maxillary expansion: a posteroanterior cephalometric evaluation. Am J Orthod Dentofacial Orthop 2002;121:129-35.

6. Inoue $\mathrm{H}$. Radiographic observation of rapid expansion of human maxilla. Bull Tokyo Med Dent Univ 1970;17:219-29.

7. Garib DG, Henriques JFC, Janson G, De Freitas MR, Coelho RA. Rapid maxillary expansion-tooth tissue-borne versus toothborne expanders: a computed tomography evaluation of dentoskeletal effects. Angle Orthod 2005;75:548-57.

8. da Silva Filho OG, Lara TS, da Silva HC, Bertoz FA. Post expansion evaluation of the midpalatal suture in children submitted to rapid palatal expansion: a CT study. J Clin Pediatr Dent 2006;31:142-8.

9. Lione R, Ballanti F, Franchi L, Baccetti T, Cozza P. Treatment and posttreatment skeletal effects of rapid maxillary expansion studied with low-dose computed tomography in growing subjects. Am J Orthod Dentofacial Orthop 2008;134:389-92.

10. Shapurian T, Damoulis PD, Reiser GM, Griffin TJ, Rand WM. Quantitative evaluation of bone density using the Hounsfield index. Int J Oral Maxillofac Implants 2006;21:290-7.

11. Martinez H, Davarpanah M, Missika P, Celletti R, Lazzara R. Optimal implant stabilization in low density bone. Clin Oral Implants Res 2001;12:423-32.

12. Baccetti T, Franchi L, McNamara JA. The cervical vertebrae maturation (CVM) method for the assessment of optimal treatment timing in dentofacial orthopedics. Semin Orthod 2005;11:119-29.

13. Cozza P, Giancotti A, Petrosino A. Rapid palatal expansion in mixed dentition using a modified expander: a cephalometric investigation. J Clin Orthod 2001;28:129-34.

14. Ballanti F, Lione R, Fanucci E, Franchi L, Baccetti T, Cozza P. Immediate and post-retention effects of rapid maxillary expansion investigated by computed tomography in growing patients. Angle Orthod 2009;79:24-9.

15. Dahlberg G. Statistical methods for medical and biological students. New York: Interscience Publications; 1940. 